\title{
Die Witterungsbeobachtungen im fränkisch-bayerischen Raum aus dem ersten Drittel des 16.Jahrhunderts unter besonderer Berücksichtigung der Aufschreibungen des Regensburger Weihbischofs Dr. Peter Krafft von 1503 bis 1529
}

\author{
Von Fritz Klemm ${ }^{1}$
}

Die erhaltengebliebenen und heute verfügbaren, vielfach unter astrologischen Vorstellungen stehenden oder entstandenen meteorologischen Beobachtungen der sogenannten vorinstrumentellen Ära, die sich in Deutschland bis zum Jahre 1678 erstreckt, da die ersten bekannten Temperaturmessungen ab 1679 in Kiel auf Arbeiten des Professors der Mathematik an der Universität Dr. Samuel Reyher (1635-1714) zurückgehen ${ }^{10,}{ }^{21}$, während Niederschlagsmessungen erst ab 1715 zu Ulm durch den Prediger am Münster und Professor der Mathematik am Gymnasium David Algöwer (1678-1737) nachweisbar sind ${ }^{1,10}$, wurden lange Zeit zwar wohlwollend, aber immerhin als eine Art Kuriosität betrachtet, der ein wirklicher meteorologischer Wert kaum zuzubilligen sei. Diese frühere Beurteilung ist längst revidiert, und eine realistische Auffassung hat den tatsächlichen Wert und die hohe praktische Bedeutung dieses alten Beobachtungsmaterials für die Klimaforschung, insbesondere alle Untersuchungen von Klimaschwankungen und Klimaperioden der letzten Jahrhunderte, sehr eindeutig erkannt und nachgewiesen. Als Beispiele seien hier stellvertretend nur zwei Arbeiten genannt, nämlich die Veröffentlichungen von Flohn, «Klima und Witterungsablauf in Zürich im 16. Jahrhundert " ${ }^{4}$ und von Lenke: «Klimadaten von 1621-1650 nach Beobachtungen des Landgrafen Hermann IV. von Hessen (Uranophilus Cyriandrus)». ${ }^{16}$

Endlich muß auf die vielfältigen Verflechtungen der vorinstrumentellen Witterungsbeobachtungen mit kulturhistorischen und historischen Aspekten hingewiesen werden, die dem gesamten Fragenkomplex einen zusätzlichen und ganz besonderen Reiz verleihen.

Wie kein anderes Gebiet Mitteleuropas hat uns der fränkisch-bayerische Raum ein ungewöhnlich reiches Erbe an derartigen Witterungsaufzeich-

1 Anschrift: F.Klemm, 600 Frankfurt am Main, Mainkai 18. 
nungen für das erste Drittel des 16. Jahrhunderts hinterlassen und damit die Grundlage der Kenntnisse des Witterungsverlaufes des fraglichen Zeitraumes geliefert ${ }^{11,29}$.

Fraglos hat sich hierbei die Ausstrahlungskraft der Blüte des kulturellen Lebens ausgewirkt, welche die Reichsstadt Nürnberg an der Wende des 15. zum 16. Jahrhundert erlebte. Auf der Grundlage eines gesicherten Wohlstandes, erwachsen aus einer festen sozialen und rechtlichen Ordnung, einem ausgedehnten internationalen Handel, nicht zuletzt aber auch dem Fleiß des sich durch hervorragende Leistungen auszeichnenden Gewerbes, war die Stadt zu einem der führenden Zentren des Humanismus und der Renaissance in Deutschland, ja Mitteleuropa geworden ${ }^{3,28,29}$.

In Nürnberg ließ sich nach seinen Aufenthalten in Italien und Ungarn im Jahre 1471 der berühmte Humanist, Mathematiker und Astronom Johannes Müller aus Königsberg in Franken, genannt Regiomontanus (1436 bis 1476), Schüler des Wiener Gelehrten Georg von Peuerbach (1423-1461), nieder, richtete sich eine Sternwarte und Druckerei ein und stellte, unterstützt von seinem Freund, dem wohlhabenden Kaufmann Bernhard Walther (1430-1504), mit eigenen und NürnbergerInstrumenten astronomische Beobachtungen und Berechnungen an, die 1474 zum Druck seiner Ephemeriden, Tafeln der Planetenstellungen für die Zeit von 1475 bis 1506, führten. Ihr Wert und ihre Beliebtheit für die außerordentlich verbreitete Fachastrologie kommt in den $28 \mathrm{im}$ Zeitraum von 1474 bis 1539 erschienenen Auflagen zum Ausdruck. Darüber hinaus ist bezeugt, daß sie sich auch in den Händen der großen Entdecker Christoph Kolumbus (1447-1506) und Amerigo Vespucci (1451-1512) befanden und auf den großen Seefahrten dieser Männer als Hilfsmittel für die astronomische Navigation benutzt wurden.

Nach Regiomontans frühem Tod am 6.7.1476 in Rom - er war dorthin zur Mitarbeit an der notwendigen Kalenderreform berufen worden - setzte Bernhard Walther mit dem befreundeten Maler und Graphiker Albrecht Dürer (1471-1528) die astronomische Arbeit in Nürnberg fort, so daß eine Serie moderner Messungen verfügbar war, als Nikolaus Kopernikus (1473 bis 1543) seine astronomische Arbeit begann, die bekanntlich in der Großtat der Schaffung eines neuen Weltbildes gipfelte ${ }^{28,}{ }^{29,30}$.

In Nürnberg krönte 1487 Kaiser Friedrich III. (1415-1493) während eines längeren Aufenthaltes in der Stadt den «Erzhumanist» Conrad Celtis (1459-1519) zum Dichter; aus Nürnberg stammte Propst Melchior Pfinzing (1481-1535), der als Geheimschreiber des Kaisers Maximilian I. (1459-1519) den Theuerdank verfaßte; der Nürnberger Martin Behaim (1460-1506) 
machte Regiomontans Erkenntnisse für die portugiesische Seefahrt nutze und wurde berühmt, als er, wegen einer Erbschaftsangelegenheit in seiner Vaterstadt weilend, für den Rat der Stadt einen «Erdapfel» mit den neuesten Entdeckungen herstellte. Für diesen ältesten erhaltenen, endgültig im Jahre 1494 fertiggestellten Erdglobus zahlte die Stadt den nicht geringen Preis von 28 Gulden.

In Nürnberg gab der Mathematiker, Astronom und Propst an der Marienkirche Konrad Heinfogel $(\dagger 1517)$ in Zusammenarbeit mit dem Professor der Mathematik Johann Stab (†1522) und Albrecht Dürer im Jahre 1515 die ältesten gedruckten Sternkarten sowohl des nördlichen als auch des südlichen Himmels heraus; der Schlossermeister Peter Henlein (1480-1542) baute von 1510 an kleine «selbgeende» Reise- und Halsuhren mit Federzug und 40 stündiger Laufzeit, die als staunenswerte Neuerung vom Rat der Stadt an hohe Persönlichkeiten verschenkt wurden, und endlich war der einer reichen, ratsfähigen Familie entstammende Sammler, Altertumsliebhaber und Sprachkenner Willibald Pirkheimer (1470-1530), der mit den führenden Gelehrten seiner Zeit einen ausgedehnten Briefwechsel pflegte und wegen seiner Gastfreundlichkeit sehr geschätzt wurde, der Mittelpunkt des humanistischen Kreises in Nürnberg. Seine astronomischen Studien befaßten sich vorwiegend mit dem beliebten Gebiet der Sterndeutung, d.h. der Stellung von Horoskopen. Hierzu gibt es eine kennzeichnende Anekdote: Bei einer Auseinandersetzung mit einer Dame, deren Sohn er im Jahre 1506 nicht anerkennen will, ist Pirkheimer schnell beruhigt, denn aus dem Horoskop des Kindes geht hervor, daß er nicht lange leben wird ${ }^{3,}{ }^{29}$.

Es ist evident, daß sich das meteorologische Interesse in erster Linie auf die ausführlichen täglichen Witterungsbeobachtungen konzentriert, die im Gegensatz zu chronistischen Aufzeichnungen nicht nur besonders auffällige Elementarereignisse verzeichnen, sondern das für jede wissenschaftliche Auswertung erforderliche «normale Wetter» erfassen.

Dem Zeitgeist entsprechend, waren die Verfasser zumeist mehr oder weniger Anhänger der aus dem Altertum überkommenen astrologischen Lehre vom Jahresregiment der sieben Planeten, deren sich jeweils für ein Jahr abwechselnder Einfluß angeblich alle irdischen Vorgänge und damit auch das Wetter mit allen Folgen bestimmte. Aus dem Glauben, daß es möglich sei, außer atronomischen Ereignissen auch die Witterung für ein ganzes Jahr voraus zu berechnen, entwickelte sich die weit verbreitete Literatur der Praktiken und Prognostiken, die in der zweiten Hälfte des 16. Jahrhunderts den Höhepunkt ihrer Popularität erreichte. Die Stadt 
Nürnberg war eines der Zentren der kommerziellen Ausnutzung dieses astrologischen Irrglaubens, bei der allerdings ohne jedes Risiko viel Geld verdient werden konnte.

Über die Gründe, die zu den frühen regelmäßigen täglichen Witterungsaufzeichnungen führten, schrieb Hellmann ${ }^{11}$ : «Viele Praktikenschreiber, von denen manche als berufsmäßige Vertreter der Astrologie an den Universitäten wirklich gelehrte Männer waren, nahmen unwillkürlich Veranlassung, das Wetter, das sie alljährlich voraussagten, auch wirklich zu beobachten und ein regelmäßiges meteorologisches Tagebuch zu führen, zumeist in der Absicht, die von ihnen vertretenen astrometeorologischen Theorien zu stützen bzw. zu berichtigen». Zumindest ein Teil der alten täglichen Wetterbeobachtungen muß daher als eine Art «Prognosenkontrolle» betrachtet werden.

Für die Witterungsnotizen selbst verwendete die Mehrzahl der vorinstrumentellen Beobachter des fraglichen Zeitraums «Stöfflersche Almanache», eine Fortsetzung der Ephemeriden des Regiomontanus, da diese sich wegen des darin verfügbaren Raumes besonders für fortlaufende tägliche meteorologische oder überhaupt tagebuchartige Eintragungen eigneten.

Der Tübinger Professor der Mathematik Johann Stöffler (1452-1531) wurde vor allem durch seine vorstehend genannten, die Zeitspanne von 1499 bis 1531 umfassenden Ephemeriden bekannt: «Almanach nova plurimis annis venturis inserventia per Joannem Stoefflerinum Justingensem et Jacobum Pflaumen Ulmensem accuratissime supputata et toti fere Europae dextro sydere impartita, Ulm bei Johann Reger 1499 ». Es existiert eine größere Zahl verschiedener Ausgaben dieses geschätzten Almanachs, dessen spätere Herausgeber die Italiener L. Gauricus und P.Pitatus waren. Auch die Ephemeridenwerke von Stadius, Carelli, Origanus und anderen wurden zu Witterungseintragungen benutzt.

Für das erste Drittel des 16. Jahrhunderts stehen uns heute zwei Beobachtungsreihen mit täglichen Witterungsaufzeichnungen aus dem fränkischbayerischen Raum zur Verfügung, und zwar handelt es sich um die kurze, die Jahre 1510-1511 umfassende, in München durchgeführte Beobachtungsreihe des berühmten bayerischen Humanisten, Historiographen und Prinzenerziehers Johann Turmair, genannt Aventinus (1477-1534) ${ }^{15}$, die sich in einem Exemplar des Stöfflerschen Almanach nova, Ulm 1499 der Staatsbibliothek München, Signatur 4 L impr. n.c.mss. 56 befindet, und zweitens um die fast 19jährigen meteorologischen Beobachtungen von hervorragender Qualität, die der bedeutendste Prior des Augustinerchorherrenstiftes 
Rebdorf bei Eichstätt, Kilian Leib, in dem Zeitraum von 1511 bis 1531 angestellt und ebenfalls in einen Stöfflerschen Almanach nova, Ulm 1499, eingetragen hat, der im Besitz der Staatsbibliothek München ist und die Signatur 4 L impr.n.c.mss. 73 hat ${ }^{13,20}$.

Als wichtige Ergänzungen hierzu müssen die erhaltengebliebenen Witterungsnotierungen mit unregelmäßigen oder unvollständigen Angaben betrachtet werden. Nicht, daß ihre Bedeutung auch nur annähernd den regelmäßigen täglichen Beobachtungen des Aventinus oder Kilian Leibs gleichkäme; ihr Wert liegt insbesondere darin, daß bekannte Wettergeschehnisse bestätigt oder ergänzt, aber auch bisher unbekannte Fakten neu erschlossen werden.

Es ist kein Zufall, daß sich die Mehrzahl dieser fragmentarischen Witterungsaufzeichnungen auf die Stadt Nürnberg und den benachbarten fränkischen Raum beziehen.

Bereits aus dem Jahre 1499 liegen Aufschreibungen des Astronomen, Magisters und Vorstehers (propositus) der Marienkirche zu Nürnberg Konrad Heinfogel vor, der zu dem Humanistenkreis um Willibald Pirkheimer gehörte und, wie erwähnt, unter Mitarbeit des kaiserlichen Mathematikers Johannes Stab und Albrecht Dürers im Jahre 1515 die ältesten gedruckten Sternkarten des ganzen Himmels herausgab und dadurch Berühmtheit erlangte. Seine Witterungsangaben sind enthalten in einem Stöfflerschen Almanach nova, Ulm 1499, der Staatsbibliothek Bamberg unter der Signatur Inc.typ. H.IV. $21^{11,}{ }^{24,} 29$.

Die Ergänzungsreihe setzt sich fort mit den gelegentlich auch regelmäßig ausgeführten Witterungsbeobachtungen von 1499 bis 1506 des aus Karlstadt in Unterfranken stammenden Astronomen, Mathematikers und Predigers Johannes Schöner (1477-1547), der etwa ab 1515 in Bamberg in seiner eigenen Druckerei mathematische, astronomische und astrologische Werke druckte oder in verschiedenen Nürnberger Druckereien anfertigen ließ, Erd- und Himmelskugeln herstellte, vermutlich auch Sonnenuhren baute und später, ab 1526, als Professor der Mathematik am Gymnasium zu Nürnberg wirkte.

Die Wetterbeobachtungen Johann Schöners verteilen sich, seinem beruflichen Werdegang nach Abschluß der Studien in Erfurt entsprechend, in der Hauptsache auf drei Orte. Am 22.2.1499 trat er sein erstes Amt als Lehrer in Gemünden in Unterfranken an, am 18.4.1501 erfolgte nach Reisen nach Erfurt und Bamberg die Ernennung zum Kaplan in Hallstatt bei Bamberg, und am 4.6.1504 wurde er als Vikar in seine Vaterstadt Karlstadt 
versetzt, wo er, abgesehen von einem Aufenthalt in Bamberg vom 4.5.1506 bis 29.10.1506, bis zum Ende seiner meteorologischen Tätigkeit verblieb. Die Aufzeichnungen selbst wurden in einen Band der Ephemeriden des Regiomontanus eingetragen, der jetzt Eigentum der National-Bibliothek Wien ist und die Signatur Inc. IV.H. 7 trägt.

In diesem Bande sind übrigens außer Schöners Beobachtungen auch frühere Witterungsaufzeichnungen enthalten, nämlich die Bamberger Beobachtungen von 1481-1486 des Chorherren Dr. Antonius von Rotenhan $(† 1498)$, letzter Stammhalter der Ebelsbacher Linie und Neffe des Bamberger Bischofs Anton von Rotenhan (†1469) und die Nürnberger Beobachtungen von 1487 des Kaufmanns und Freundes Regiomontans Bernhard Walther. Hinzu kommen geschichtliche Aufzeichnungen für 1488 und ein Verzeichnis der Preise für Wein und Getreide von 1490 bis 1492 eines Unbekannten $^{10,11,23,29}$.

Wiederum Nürnberger Witterungsbeobachtungen führte in den Jahren 1513-1520 der mit Pirkheimer und dessen Kreis im Verkehr stehende Pfarrer an der Kapelle St. Johann, Mathematiker und Astronom Johannes Werner (1468-1522) aus, der sich sehr viel mit Astrologie beschäftigte, das Schicksal auf Grund von Horoskopen wie das Wetter durch Planetenstellungen zu deuten suchte und z.B. den Kometen vom 15.8.1506 als gefährlich ansah, wie aus einem Brief des Bamberger Kanonikus Lorenz Beheim (1457-1521) vom 22.12.1506 hervorgeht. Bedauerlicherweise sind Johannes Werners meteorologische Originalaufzeichnungen verlorengegangen $9,11,29$.

Außerordentlich bemerkenswert ist die Tatsache, daß uns Johannes Werners Beobachtungen für die Zeit von 1513 bis 1515 dennoch erhalten geblieben sind, da sie posthum von Johann Schöner im Jahre 1546 veröffentlicht wurden unter dem Titel: «Canones sicut brevissimi, ita etiam doctissimi, complectentes praecepta et observationes de mutatione aurae clarissimi mathematici Joannis Verneri. Norimbergae 1546.»

Es handelt sich hier um die ersten durch Druck veröffentlichten deutschen Wetterbeobachtungen. Ergänzend sei vermerkt, daß das älteste deutsche Druckwerk über das Wetter überhaupt, Leonhard Reynmanns 1505 erschienenes: «Wetter Biechlein. Von warer erkantnus des wetters, Also das ain yeder er sey gelert oder vngelert, durch alle natürliche anzeigung die meinung des wetters ... wissen vnd erkennen mag ...» s. 1 . so beliebt und geschätzt war, daß allein in den Jahren 1510-1514 mehrere Auflagen in München, Augsburg und Erfurt notwendig wurden, die Nach- 
frage befriedigen zu können. Übrigens hat Hellmann die Münchener Auflage von 1510 neu herausgegeben.

Aus dem Jahre 1519 liegen Witterungsnotizen vor, die nach Hellmann ${ }^{11}$ von einem unbekannten Kleriker des Klosters Ursberg, LK Krumbach in einem Stöfflerschen Almanach nova, Ulm 1499, vermerkt wurden, der sich in der Stadtbibliothek Augsburg befindet.

Gelegentlich einer Rückfrage nach der Signatur dieses Bandes teilte die Staats- und Stadtbibliothek Augsburg im August 1969 mit, daß dort zwei Exemplare des Stöfflerschen Almanach nova, Ulm 1499 vorhanden seien, die beide die Signatur $4^{\circ}$ Ink. 596 haben und handschriftliche Rand- und Interlinearglossen aufweisen. Ein Exemplar enthält astronomisch-mathematische Einträge, in dem zweiten Band hat offenbar ein Landwirt Ereignisse aus dem bäuerlichen sowie dem Familienleben verzeichnet, während astronomische bzw. astrologische Zeichen und Angaben zur Witterung mit sehr flüssigem Duktus von eindeutig anderer Hand auf den von Hellmann erwähnten unbekannten Kleriker hinzudeuten scheinen.

Endlich kommen hinzu die in der meterologischen Literatur nur wenig beachteten Witterungsnotierungen, die sich in den Tagebuchaufzeichnungen des Regensburger Weihbischofs Dr. Peter Krafft befinden ${ }^{17}{ }^{25}$. Sie sind enthalten in einem Stöfflerschen Almanach nova, Ulm 1499, der Universitäts-Bibliothek München. Wegen der handschriftlichen Eintragungen wurde die fragliche Inkunabel $4^{\circ}$ Inc. lat. 318 dort zu den Handschriften gestellt und erhielt die neue Signatur: 4\% Cod. ms.942 (Mitteilung der Universitäts-Bibliothek München vom 3.6.1969).

Es entspricht dem Tagebuchcharakter, daß Peter Krafft keine fortlaufenden täglichen Witterungsaufzeichnungen vornahm, sondern nur besonders auffällige und markante Elementarereignisse wie Stürme, Gewitter, starke Regen- und Schneefälle, Überschwemmungen, Frühjahrsfröste, Frostbeginn- und -ende im Winter usw. beachtete. Gleichwohl muß der Wert derartiger chronistischer Wetterbeobachtungen um so höher veranschlagt werden, je seltener solches Material aus dem ersten Drittel des 16. Jahrhunderts erhaltenblieb und heute verwendet werden kann.

Nicht, daß Peter Kraffts Tagebuchblätter dem Historiker neue Aufschlüsse aus der Frühzeit der Reformation vermittelten; aber gerade solche tagesgeschichtlichen Aufzeichnungen eines Mannes, der fast dreißig Jahre vor und zur Zeit der bedeutendsten kirchlichen Umwälzung der neueren Geschichte als Weihbischof eines großen deutschen Bistums gewirkt hat, vermögen als wertvolle Quelle oft wirksam die Hintergründe der Ereignisse 
jener Zeit aufzuhellen, zumal dann, wenn der Berichterstatter durch seine Lebensstellung in Beziehung zu wichtigen Vorgängen und bedeutenden Persönlichkeiten seiner Zeit stand. Im übrigen tritt uns auch der Mensch Peter Krafft in seinem Tagebuch deutlich mit allen seinen Vorzügen, aber auch Schwächen entgegen.

Peter Krafft wurde als zweiter Sohn des herzoglich bayerischen Rates Dr. Peter Krafft, der am Hofe Herzogs Georg des Reichen (1479-1503) eine einflußreiche Stellung einnahm, während eines vorübergehenden Aufenthaltes der Familie zu Blaubeuren um 1470 geboren. Über seine Jugendjahre sind keine Einzelheiten bekannt. Belegt ist, daß er am 21.10.1491 mit seinem älteren Bruder Hieronymus, über dessen späteres Schicksal keine Angaben vorliegen, die Hochschule in Ingoldstadt bezog, bezeugt ist ferner, daß die beiden Brüder - es handelte sich um einen besonderen Gunstbeweis des Herzogs ihrem Vater gegenüber - bei der Immatrikulation keine Aufnahmegebühr zu bezahlen brauchten.

Peter Krafft widmete sich dem Studium der philosophischen, juristischen und theologischen Fächer, und es ist symptomatisch, daß der berühmte Humanist Konrad Celtis, der im gleichen Winterhalbjahr 1491/92 in Ingolstadt seine Lehrtätigkeit aufnahm, auf ihn offenbar keinerlei Einfluß gewonnen hat. Bereits am 6.4.1493 zum Priester geweiht (Cod. lat. 26297 der Staats-Bibliothek München), schloß Peter Krafft die Ingolstädter Studienzeit mit der Erlangung der philosophischen Magister- und der juristischen Doktorwürde am 30.9.1500 ab.

Aber schon vorher hatte der Wittelsbacher Rupert II., Bischof von Regensburg von 1492 bis 1507 - vermutlich hatte Herzog Georg der Reiche seine Hand im Spiele -, den einer fremden Diözese angehörigen Geistlichen Peter Krafft am 4.8.1500 zum Weihbischf von Regensburg als Nachfolger des am 31.7.1500 verstorbenen bisherigen Weihbischofs Johannes vorgeschlagen und zugleich den mit Regensburg eng verbundenen KardinalDiacon von Siena, Francesco Piccolomini, den späteren Papst Pius III. (1503), um Empfehlung des neuen Weihbischofs beim Papst Alexander VI. (1492-1503) gebeten.

Ende Dezember 1500 trat demgemäß Peter Krafft die Reise nach Rom an, erreichte am 27.12.1500 Innsbruck, am 31.12.1500 Bozen und traf endlich am Ziel der Reise am 20.1.1501 ein. Hier empfing er bereits wenige Tage später, d.h. am 27.1.1501 die päpstliche Bestätigung zum Weihbischof von Regensburg, am 14.2.1501 erfolgte seine feierliche Weihe zum Bischof von Hierapolis in Phrygien, und am 19.2.1501 konnte die Heimreise 
angetreten werden. Nach einem Dankbesuch in Siena bei dem Kardinal Piccolomini kam der neue Weihbischof Peter Krafft am 21.3.1501 in Regensburg an, wo er am 30.3.1501 die festliche Übertragung des neuen Amtes durch Bischof Rupert II. erlebte.

Zugleich erhielt er die Einkünfte der Pfarrei Alteglofstein, die zum bischöflichen Tafelgut gehörte und dem jeweiligen Weihbischof übertragen wurde.

Zur Zeit des Amtsantritts des Weihbischofs Peter Krafft stand die Stadt Regensburg - im Gegensatz zu der blühenden Stadt Nürnberg - vor dem wirtschaftlichen Ruin. Der einst so bedeutende Handel der Freien Reichsstadt war im 15. Jahrhundert ständig zurückgegangen, weil vor allem verschiedene äußere Umstände eine Verlagerung des Welthandels und der Handelswege mit sich gebracht hatten. Die Wege aus Italien nach Norden führten nunmehr über Augsburg, Ulm und Nürnberg. Auch die Reichsfreiheit war kein Segen für die Stadt, deren Territorium auf engstem Raum eingeschlossen und von bayerischem Gebiet umgeben war. Das erklärt auch, warum sich der bayerische Herzog Albrecht IV. der Weise (1465-1508), der erste moderne Staatsmann unter dem bayerischen Herzogshut, auf eine starke Anhängerschaft stützen konnte, als er sich im Jahre 1484 der Stadt bemächtigte. Es schien, als sollte Regensburg wie vor 1245 wieder bayerische Hauptstadt werden, da Albrecht IV. bereits mit Nachdruck den Bau eines Schlosses und die Errichtung einer Universität betrieb.

Kaiser Friedrich III. vereitelte jedoch die Pläne des Wittelsbachers und erzwang im Jahre 1492 den Abzug des Herzogs und die Herausgabe der Reichsstadt ${ }^{3}$.

Es waren in der Tat keine günstigen Auspizien, unter denen die fast 30 jährige Amtstätigkeit Peter Kraffts begann, in deren Verlauf er infolge seiner zahlreichen und weiten Reisen alle Teile des umfangreichen Kirchensprengels wie kaum ein anderer kennenlernte, mit einem großen Teil der weltlichen und klösterlichen Geistlichkeit des Regensburger Bistums in Berührung kam und vor allem auch mit den kirchlichen Begebenheiten der Stadt Regensburg aufs engste verbunden war. Das galt um so mehr, als nach dem Tode Bischofs Rupert II. am 19.4.1507 der erst 19jährige Wittelsbacher Johann, Pfalzgraf bei Rhein und Herzog von Bayern, am 13.12.1507 als neuer Oberhirte des Bistums Regensburg inthronisiert wurde. Als das Regensburger Domkapitel ihn nach dem Tode seines Vetters zum Bischof erkor, hatte man die großen politischen Schwierigkeiten des Hochstifts im Auge, die ein Fürst mit mächtigen Verwandten noch am ehesten 
bewältigen konnte. Die römische Kurie berücksichtigte solche Erwägungen und bestätigte die Postulation der Domherren mit Bulle vom 27.10.1507, nach welcher der neue Administrator bzw. Bistumsverwalter mit dem 27.Lebensjahr die Bischofsweihe empfangen könne und solle, eine Verpflichtung, die dieser aber nie erfüllte. Da der Administrator also keine geistlichen Weihen besaß, mußte Weihbischof Peter Krafft Firmungen und Priesterweihungen vollziehen - er hat allein 6 Äbte und die Äbtissin von Geisenfeld geweiht -, bei hohen Festen das Hochamt halten und bei besonderen Gelegenheiten die kirchlichen Feierlichkeiten vornehmen 5, 12, 25, 26.

Nachdem Peter Krafft im Sommer 1501 zu Gunsten eines Verwandten auf eine Chorherrenstelle bei St. Johann verzichtet hatte, verlieh ihm der Administrator Johann am 14.1.1508 die Anwartschaft auf die nächste Chorherrenpfründe an der Alten Kapelle, und bereits am 24.4.1508 wurde die verheißene Stelle frei. Zu den Zeugen der Aufschwörung des Kanonikus Peter Krafft gehörte u.a. auch sein Verwandter, der Magister und Domscholastikus zu Regensburg Silvester Krafft. Am 26.5.1510 wurde Peter Krafft zu Sitz und Stimme im Stiftkapitel zugelassen, am 22.10.1520 erhielt er die Pfarrei Oberdolling bei Ingolstadt und am 15.6.1521 auch die Pfarrei Weiden in der Oberpfalz, an der er aber wenig Freude erlebte, da sein Vikar Ulrich Hanauer seines Amtes schlecht waltete und sich vor allem Johann Freysleben seit 1522 in der Stadtpfarrkirche St. Michael offen zu Luther bekannte, wobei er die Priester der alten Kirche als «Herrgottsverkäufer und Tempelknechte» bezeichnete. Im Dezember 1523 vor das geistliche Gericht nach Regensburg geladen, verlangte Freysleben die $\mathrm{Zu}$ sicherung freien Geleits und in seiner gleichzeitigen beißenden Schmähschrift gegen die Ingolstädter Professoren, die die neue Lehre bekämpften, hieß es von Peter Krafft, er habe zu Weiden gepredigt: "Was Glaube sei, brauche man nicht zu wissen, das müsse man den großen Hansen (ein damals beliebtes Schlagwort) überlassen!»

Stark beeinflußt wurde die Arbeit des Weihbischofs Peter Krafft durch die politischen Wirren jener Zeit. Nur mit tiefster Anteilnahme und Erschütterung erblickte er auf seinen ausgedehnten Reisen die furchtbaren Folgen und Verwüstungen, die der unselige Landshuter Erbfolgekrieg von 1503 bis 1505 in den Gebieten des Regensburger Bistums hervorgerufen hatte. Dieser bayerische Bruderkrieg, in dem u.a. Götz von Berlichingen bei der Belagerung von Landshut seine rechte Hand verlor, entbrannte zwischen den Wittelsbacher Linien Pfalz und München um das Erbe der mit Herzog Georg dem Reichen ausgestorbenen Landshuter Linie und wurde durch den Schiedsspruch Kaisers Maximilian I. beendet. Im Jahre 1506 erfolgte dann unter Herzog Albrecht IV. die Wiedervereinigung der vier wittelsbachischen Teilterritorien München, Landshut, Ingolstadt und Straubing zu 
einem bayerischen Gesamtstaat und der Erlaß des Primogeniturgesetzes zur Vermeidung neuer Landesteilungen ${ }^{14,25}$.

Im Zuge des Wiederaufbaues in den hart betroffenen Gebieten der Diözese Regensburg erließ der Administrator Johann 1508 eine Verordnung, die den verantwortlichen Stellen dringend die Weihung der Kirchen und Altäre ans Herz legte und auch der üblichen Auslagevergütung gedachte: «Wenn es in den verflossenen Jahren erlaubt war, daß in entweihten oder noch nicht geweihten Kirchen und Kapellen Gottesdienste abgehalten wurde, so gründete sich diese Erlaubnis doch auf die Voraussetzung, daß die Weihungen sobald als möglich nachgeholt würden; deshalb bestimmen wir jetzt, daß der hochwürdige Weihbischof Petrus alle entweihten Kirchen besuchen und weihen, auch die Kirchengeräte einer eingehenden Besichtigung unterziehen soll und für seine Mühen und Auslagen eine gebührende Entschädigung beanspruchen darf.»

Diese erwähnten Gebühren stellten viele Jahre eine erhebliche Einnahmequelle dar, deren etwaiger Verlust höchst schmerzlich empfunden werden mußte. Gerade dieser Umstand trat aber im Jahre 1524, gelegentlich der Reformationsversuche ein, die bei einer Tagung katholischer Fürsten in Regensburg beschlossen wurden. Es sollte u.a. auch das weihbischöfliche Amt von dem Makel der unwürdigen Gelderhebungen befreit werden, die sich allmählich hier und da eingeschlichen hatten. In der «Regensburger Reformation» wurde daher dekretiert: "Wir setzen und ordnen auch, daß durch den Weihbischof von Weihung der Kirchen und Altäre, wie dann die geistlichen Rechte wollen, außerhalb der Procuration gar nichts genommen werde, denn es gebührt sich, daß die Ordinari von ihrem bischöflichen Einkumen die Weihbischof nach Ordnung des päpstlichen Stuhls und ihres Standes Gelegenheit versehen und zu Unwürden bischöflichen Stands nichts unziemlichs fürgenommen werde $»{ }^{6}$

Peter Krafft, der die Regensburger Reformationsversuche mit zunehmendem Mißtrauen verfolgt hatte, brachte seine Empörung über den ihm unannehmbar erscheinenden Beschluß in seinem Tagebuch mit schöner Offenheit zum Ausdruck: «Daß doch diese Versammlung nie stattgefunden hätte.» Seinen Versuchen, die unbequeme Bestimmung zu umgehen und hierzu den Schutz der weltlichen Obrigkeit in Anspruch zu nehmen, blieb der Erfolg aber trotz empfehlender Schreiben der Bischofsadministratoren von Regensburg und Passau versagt.

Die revolutionären Unruhen, welche die Reichsstadt Regensburg in der Zeit von 1511 bis 1513 erschütterten, betrafen den Administrator Johann und den Weihbischof Peter Krafft kaum, wenngleich sie sich als geistliche Oberhirten bemühten, Frieden und Versöhnung zu stiften. An der Tatsache, daß das nicht gelang, läßt sich schon hieran der Autoritätsverlust der Kirche am Vorabend der Reformation erkennen ${ }^{5,7,12,25 .}$

Ursache der Kämpfe war die allgemeine Unzufriedenheit mit dem kaiserlichen Reichshauptmann. Nicht, daß dessen Person angefeindet wurde; es war vielmehr die hohe Besoldung, die für ihn aufzubringen war und von der Bürgerschaft um so überflüssiger empfunden wurde, als durch die häufige Abwesenheit des kaiserlichen Beauftragten die der Stadt entzogenen Regierungs- und Verwaltungsaufgaben häufig in nicht vertretbarer Weise verzögert wurden. Die Stadt setzte daher ihren ganzen Einfluß ein, den Kaiser zu bewegen, die Stelle des 1511 verstorbenen Reichshauptmanns Sigmund von Rohrbach nicht erneut zu besetzen. Gegen alle Einsprüche übertrug jedoch Maximilian I. diese Pfründe seinem Günstling, dem Rat Ritter Thomas Fuchs von Schneeberg, und bedachte ihn mit vierhundert Gulden, welche die Regensburger zu entrichten hatten. 
In einer wohlhabenden Stadt hätte die Besoldung des kaiserlichen Vertreters die Bürger wohl kaum bis zur Weißglut erhitzen und zu furchtbaren Exzessen treiben können. Als dann nach dem Ende der Pöbelherrschaft über Regensburg ein unnachsichtiges Strafgericht hereinbrach und die Haupträdelsführer, der Dombaumeister Wolfgang Roritzer, der Bildschnitzer Michael Loy und vier weitere Bürger zum Tode verurteilt und enthauptet wurden, suchten die von der wirtschaftlichen Rezession bedrängten, aber auch durch aufwendiges Leben, Liederlichkeit und Müßiggang verarmten Regensburger Händler und Handwerker natürlich nach den "wahren» Schuldigen, die sie nur allzu schnell in den Juden fanden. Auf diese konzentrierte sich nun der Haß und der Grimm der Regensburger.

Bei ihrer Schutzherrschaft, der österreichischen Regierung, beschwerten sich die Juden in der Folgezeit mehrfach darüber, daß ihnen schikanöse Ratsverordnungen mehr und mehr den Boden ihrer wirtschaftlichen Existenz entzogen, sie klagten aber auch zugleich über die Predigten, in denen man sie ständig angriff, und führten als Beweis ganze Passagen der Hetzreden an, die öffentlich von der Kanzel der Minoritenkirche herab gehalten wurden. Die antisemitische Stimmung der aufgewühlten Bevölkerung Regensburgs erfuhr auch eine Beruhigung, als einer der Haßprediger, vom Rat der Stadt zur Verantwortung gezogen, widerrufen und die Stadt verlassen mußte.

Im Jahre 1518 sah sich Kaiser Maximilian I. dann genötigt, dem Rat durch einen Herold sein Mißfallen über die Unterdrückung der Juden in Regensburg kundzutun und zu befehlen, dem Domprediger Balthasar Hubmaier (1485-1528) nicht mehr in die Stadt einzulassen. Dieser Geistliche, Schüler und Freund von Johann Eck (1486-1543), der ihn den gebildetsten Mann Europas nannte, war nach einer schnellen Karriere an der Universität Ingolstadt nach Regensburg gekommen, um hier die Stelle eines Dompredigers als Sprungbrett zu einem Kanonikat zu benutzen. Die heftige Agitation gegen die Juden, für Hubmaier vor allem ein Weg zur Popularität, hatte ihm im August 1518 eine Zitierung vor den Augsburger Reichstag eingetragen, doch die mächtigen Gönner des wortgewaltigen Demagogen, der Administrator Johann von Regensburg, die Herzoginwitwe Kunigunde von Bayern und andere Persönlichkeiten traten für ihn ein mit dem Erfolg, daß er nach Regensburg zurückkehren und seine Hetzarbeit fortsetzen durfte $2,8,25,26,27$.

Allerdings glaubten sich sowohl der spätere Führer der Täuferbewegung Hubmaier als auch der Regensburger Klerus in ihrem Kampf gegen die Juden durch eine päpstliche Bulle legitimiert und gesichert. Schon im Jahre 1512 hatte nämlich der Administrator von Regensburg ein Diözesanmandat gegen den Wucher der Juden verkünden lassen, in dem er auch die weltlichen Richter, die einen Christen zur Zahlung von Zinsen anhielten, mit dem Kirchenbann bedrohte. Auf Ansuchen hatte Papst Leo X. (1513-1521) die oberhirtliche Verordnung, die inhaltlich dem Zinsverbot des kanonischen Rechts entsprach, bestätigt. Es war daher nur folgerichtig, daß die Bulle des. Papstes, der an sich den Juden freundlich gesinnt war und an seinem Hofe jüdische Ärzte und Musiker hatte, von Balthasar Hubmaier als Autorität in seinen Predigten gegen das unglückliche Volk angeführt wurde.

Nach dem Tode des Kaisers Maximilian I. am 12.1.1519 zu Wels konnte sich der Judenhaß in Regensburg endlich ungehindert austoben. Die Saat war herangereift, der Rat der Stadt bestellte eine "spontane Volkserhebung», zu der der Administrator Johann auf das dringende Abraten des Domdechanten von Gumpenberg und des Oberst-Hofmeisters von Parsberg zwar kein offenes Einverständnis erklärte, seine und des bischöflichen Hofes wahre Gesinnung aber deutlich genug zu verstehen gab. 
Vereinbarungsgemäß erschienen also am 21.2.1519 viele Handwerker und Händler auf dem Rathaus und forderten die Vertreibung der Juden aus der Stadt wegen ihres Wuchers und der schmutzigen Konkurrenz, die sie dem Regensburger Handel und Gewerbe machten. Natürlich ließ der Beschluß des Rates nicht lange auf sich warten: das Ghetto wurde umstellt und einige Abgesandte des Rates, von den gut informierten Juden mit Wehklagen empfangen, eröffneten den Gemeindeältesten, daß ihr Gotteshaus in zwei Stunden abgebrochen würde und sie, die Juden selbst, Regensburg innerhalb von fünf Tagen verlassen müßten. Nachdem die Juden in größter Eile unter Klageliedern und rituellen Verfluchungen die Schriftrollen, heiligen Gefäße und das Archiv ihrer Gemeinde entfernt und den Raum profaniert hatten, damit er nicht von fremder Hand entweiht werde, wurde die Synagoge abgerissen, und am 28.2.1519 hatten alle Juden, mit Ausnahme des bischöflichen Hofjuden, die Donaustadt verlassen ${ }^{8}$.

Um vollendete Tatsachen zu schaffen, hatte der Domprediger Hubmaier geraten, an Stelle der Synagoge eine Marienkirche zu bauen, denn auch ein künftiger Kaiser durfte es sich bei aller Judenfreundschaft nicht erlauben, ein Muttergottesheiligtum wieder in eine jüdische Kapelle zurückzuverwandeln.

Bei der Zerstörung der Synagoge ereignete sich ein Unfall, wie er bei Abbrucharbeiten nicht ungewöhnlich ist. Der Steinmetzmeister Jakob Kern stürzte vom Gewölbe und wurde, am Boden liegend, auch noch von drei sehr schweren Steinblöcken getroffen. Zunächst auf einer Bahre heimgetragen, erschien er am Abend wieder und überwachte die weiteren Arbeiten. Die Regensburger betrachteten es indessen als ein Wunder, daß er bei dem Unfall nicht ums Leben gekommen war, und begannen, die Stätte der abgerissenen Synagoge als einen Gnadenort zu verehren. Alle Hände griffen zu, wo die allerseligste Jungfrau ihre Güte und fürbittende Macht geoffenbart hatte, um den Bau der neuen Marienkapelle zu beschleunigen. Der Administrator Johann, der Weihbischof Peter Krafft, das Domkapitel, Weltgeistliche und Ordensbrüder, vornehme Frauen und Jungfrauen, Bürger und Handwerker rissen Judenhäuser nieder, schleppten Steine und Balken und demolierten den jüdischen Friedhof. Eine unübersehbare Menschenmenge war anwesend und vergoß Freudentränen, als schon am Tage Mariä Verkündigung der Choraltar der neuen Kapelle im Beisein des gesamten Klerus vom Weihbischof Peter Krafft konsekriert werden konnte.

Der Bericht von dem Wunder, das sich in dieser Stelle vollzogen hatte, trieb eine Spätblüte mittelalterlicher Wallfahrtsfrömmigkeit, die bei sehr vielen Menschen in eine Psychose ausartete. Der Eifer des gläubigen Volkes wirkte sich nicht zuletzt in den Votivgaben und Geldspenden aus, die der Rat der Stadt vereinnahmte. Die hohen eingehenden Summen fanden teils zum Bau einer großen Wallfahrtskirche, teils aber auch zum Kampf gegen die Juden und den Bischof Verwendung, denn einerseits hatten sich die Juden durchaus noch nicht in ihr Schicksal ergeben und verursachten der Stadt durch ihre ständigen Klagen bei den Fürstenhöfen erhebliche Kosten, andererseits beanspruchte der Administrator Johann von Anfang an die Verwaltung der Wallfahrtseinkünfte und selbstverständlich.den dritten Teil davon für seine eigene Tasche. Nach langen, äußerst hart geführten Verhandlungen, in deren Verlauf sogar Luther und die römische Kurie angerufen wurden, kam es zu einem Vergleich: dem Bischof sprach man das oberhirtliche Aufsichtsrecht über die Kapelle und ihre Geistlichen, dem Rat der Stadt jedoch das wirtschaftlich einträglichere Patronatsrecht zu. Für den Verzicht an den Gefällen erhielt der Administrator den Betrag von 5400 Gulden. 


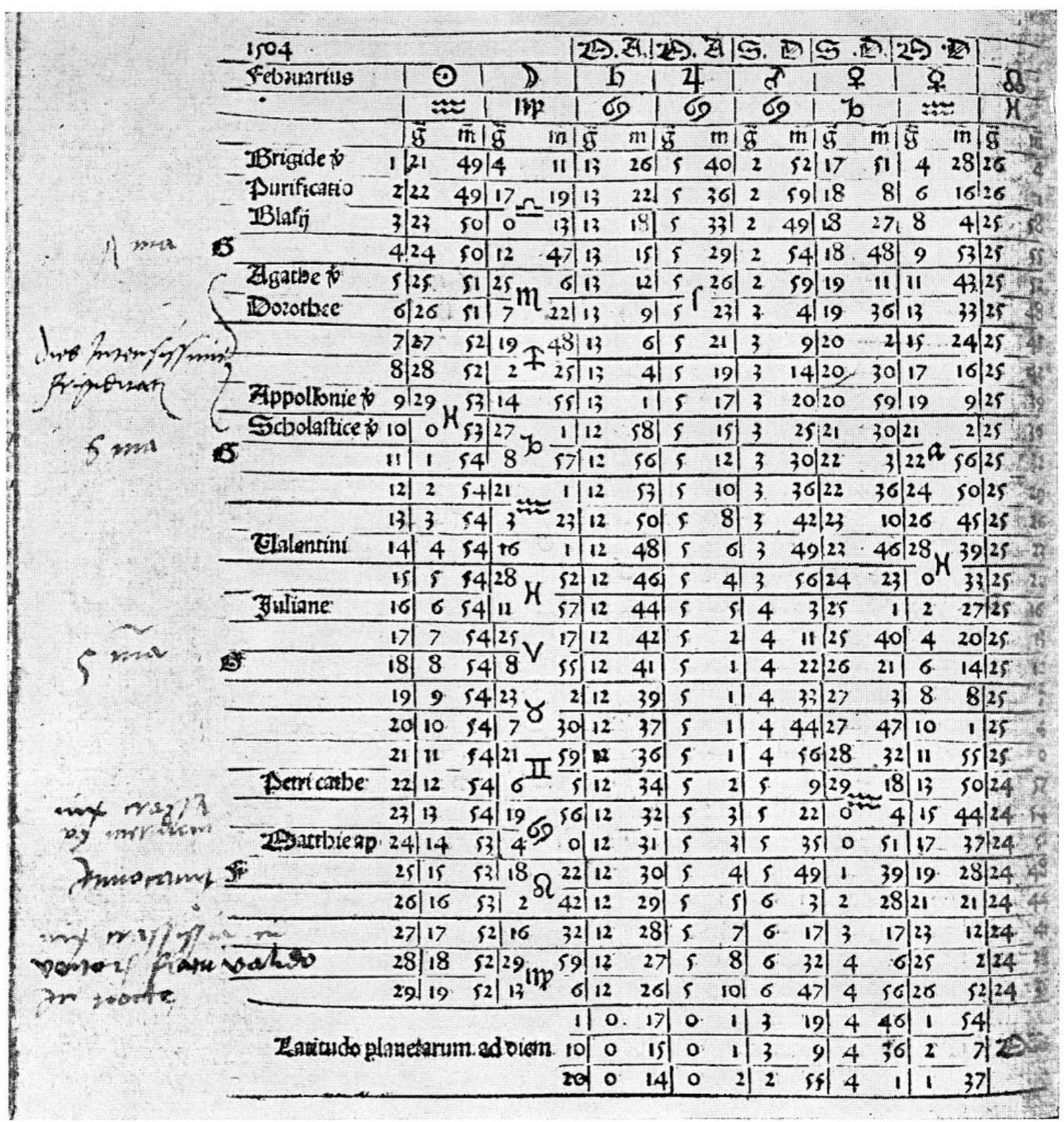

Abb. 1. Peter Kraffts Aufzeichnungen des Monats Februar 1504 in Stöfflers Almanach vova, Ulm 1499, der Universitäts-Bibliothek in München 
Die Auseinandersetzungen hatten zur Folge, daß sich die schon seit langer Zeit bestehende antiklerikale Einstellung der Regensburger Bürgerschaft erneut vertiefte. Die trügerische Einigkeit, die bei der Vertreibung der Juden aus der Stadt geherrscht hatte, war schnell wieder bitterem $\mathrm{Haß}$ gewichen, und es hatte der Geistlichkeit wenig genützt, daß sie die Mißstimmung der verarmten Handwerker und Kaufleute auf die Juden abgelenkt hatte. So war unvermeidlich, daß nun ein lange schwelendes Problem wieder auftauchte, nämlich die Steuerfreiheit des Klerus, die von der Bürgerschaft als ein wesentliches Moment der finanziellen Notlage der Stadt angesehen wurde, eine Auffassung, die schon 1514 kaiserliche Kommissare, die in der Reichsstadt eintrafen, um Ordnung zu schaffen, teilten. Im Jahre 1525 kam der Rat der Stadt endlich der Empfehlung nach, dem Klerus die Steuerfreiheit zu entziehen. Alle höheren Geistlichen Regensburgs wurden vor den Rat geladen und zur Ablegung des bürgerlichen Eides gezwungen, der sie aller bisheriger Vorrechte der Steuer- und Lastenfreiheit beraubte. Der Weihbischof Peter Krafft war der erste, der notgedrungen den Eid in die Hände des Kämmerers schwor, und nach einem zeitgenössischen Bericht soll er dabei in Tränen ausgebrochen sein, so schwer traf ihn das Vorgehen der weltlichen Obrigkeit gegen die Geistlichkeit und der damit verbundene finanzielle Verlust ${ }^{18}$.

Für den Weihbischof Peter Krafft ließen sich die folgenden Zeiten immer trüber an. Nicht nur, daß herbe finanzielle Einbußen zu beklagen waren, immer schwerer machten sich insbesondere die Auswirkungen der Reformation bemerkbar. Er, der zahllose Kirchen und Kapellen geweiht, Ablässe und Bruderschaften bestätigt, Priesterweihungen und Firmungen erteilt, alles kirchliche Handlungen, welche die neue Lehre Luthers als ungöttlich, menschlich und irrig verwarf, war zu tief mit dem Leben der alten Kirche verbunden, um in dem Auftreten des Wittenberger Augustiners etwas anderes als den verabscheuungswürdigsten Verrat sehen zu können. Offenbar gehörte Peter Krafft zu den vielen Geistlichen seiner Zeit, die nicht sehen konnten und wollten, daß vieles in der alten Kirche an innerer und überzeugender Kraft verloren hatte und nicht mehr zu halten war. Keinesfalls aber darf verkannt werden, daß er sich in seiner Stellung, seinem Lebensberuf, seinem ganzen Denken und Empfinden durch die Predigten und Schriften Martin Luthers beunruhigt, ja bedroht fühlte, zumal das Amt des Weihbischofs in den Reformationsflugschriften besonders scharf angegriffen wurde. Heißt es in dem berüchtigten «Wegegespräch gen Regensburg» schon derb genug: "Was verricht oder was schafft uns derselb an statt des Bischofs? Worinnen ist er sein Helfer? Ich mein, er hilft ihm die Bauern zu besch ... und zu schinden», so klingen in der "Klag und Antwort von lutherischen und päpstlichen Pfaffen über die Regensburger Reformation » des Jahres 1524 bereits andere Töne auf, und es wird unverhüllt mit den Dreschflegeln der Bauern gedroht: «Lieben fladenweiher, bleibt dahaim mit euren weihen, das raten wir euch» und weiter: «laßts euch gesagt sein: der pauren trischel wartet auf euch, kumpt oder bleibt aus." Begreiflicherweise mußte eine solche Sprache den der katholischen Kirche und ihren Einrichtungen treu ergebenen Weihbischof Peter Krafft zutiefst empören ${ }^{22,}{ }^{25}$.

Es blieb Peter Krafft erspart, den offenen Übertritt der Reichsstadt Regensburg zu der neuen Lehre Luthers und die Umwandlung der Wallfahrtskirche "Zur schönen Maria» in die erste evangelische Stadtkirche «Neue Pfarr» zu erleben, denn, schon längere Zeit kränkelnd, starb er am 16.3.1530 und fand unter großer Anteilnahme seine letzte Ruhestätte im Kreuzgang der Alten Kapelle.

Die noch erhaltene Grabplatte, die uns sein Bildnis in ganzer Gestalt erhalten hat, trägt 
die Inschrift: «Anno Domini 1530 die vero 16. Marcii obiit reverendus pater dominus Petrus Krafft, utriusque iuris et artium liberalium doctor, suffraganeus Ratisponensis, qui ad 30 annos suffraganeus rexit. Cuius anima Deo vivat Amen ».

Nach der Darstellung der meteorologischen Beobachtungen und Beobachter aus dem ersten Drittel des 16. Jahrhunderts im fränkisch-bayerischen Raum und dem Versuch, die Tagebuch- und Witterungsaufzeichnungen des Regensburger Weihbischofs Dr. Peter Krafft in die adäquaten kulturhistorischen und geschichtlichen Ereignisse jener Zeit zu projizieren, folgen Angaben über den von Schottenlohr ermittelten Weg, auf dem Peter Kraffts Stöfflerscher Almanach nova, Ulm 1499, in die Universitäts-Bibliothek München gelangte. Wohin das Tagebuch nach Peter Kraffts Tod kam, war nicht mehr feststellbar, aber etwas später befand es sich im Besitz des Lizentiaten der Rechte Salomon Rhebein aus Lübeck, eines eifrigen Büchersammlers, der neben juristischen Studien auch genealogische Forschungen über das Haus Anhalt betrieb. Dann wurde der Hauptnachlaß S. Rhebeins von dem Rechtsgelehrten Plato Matthias Schilher erworben, während Peter Kraffts Almanach Stöffler in der Privatbibliothek des Ingolstädter Professors der Theologie Martin Eisengrein Aufnahme fand und mit dessen hochherzigem Vermächtnis in die Universitäts-Bibliothek Ingolstadt kam. Mit dieser wurde er vorübergehend nach Landshut und von dort nach München übergeführt ${ }^{25}$.

Peter Kraffts Witterungsaufzeichnungen in dem Stöfflerschen Almanach nova, Ulm 1499, der Universitäts-Bibliothek München, Signatur $4^{\circ}$ Cod. ms. 942 enthalten, wie bereits ausdrücklich betont, für den Zeitraum von 1505 bis 1529 nur Hinweise auf besonders markante Witterungsgeschehnisse und sind damit der chronistischen Meteorologie zuzuordnen, wie die Abbildung auf Seite 77 der Original-Aufschreibungen Peter Kraffts vom Monat Februar 1504 zeigt.

Nachstehend folgt zum Schluß die vollständige Zusammenstellung Peter Kraffts Witterungsaufzeichnungen für die Zeit vom 8.6.1503 bis 25.7.1529:

1503, 8. Juni: illo die ceciderunt in Ratispona ad modum crucis et diversi super diversos homines.

1. Nov.: pluvia ad 30 dies contingit.

13. Nov.: nix primum cecidit ad noctem.

15. Nov.: congelatio magna.

1504, 9. Jan.: congelatio maxima.

24.-26. Jan.: dies frigidissimae. Congelatio Danubius.

25. Jan.: dies Pauli fuit clarissima hoc anno. 
5.-10. Febr.: dies intensissimae frigiditatis.

23. Febr.: nix crassa post meridiem.

27. Febr.: nix crassissima cum ventorum flatu valido in nocte.

5. März: iterum nix.

6. März: resolucio magna in nocte, crevit Danubius valde.

20. März: nix crassa hac nocte et Otto Hechtlin submerrus est in der Nab.

11. April: pluit.

13. April: ningit.

2. Nov.: nix primum cecidit in nocte.

1505, Jan.: novus annus valde nubilosus fuit cum diebus sequentibus.

9. Jan.: nix crassa in nocte et secunda (die) nix.

16. Jan.: nix crassissima et tertia.

19. Febr.: Nota: malum diem habui ex infirmitate.

24. Febr.: dies frigidissimae cum sequentibus hoc anno.

19. März: audivi tonitrum Ratisponae tribus vicibus hora comple torii.

17. Juli: mirabilis ad noctem illam tempestas.

1506, Jan.: dies primus admotum ventosus cum nive crassa.

1. Jan.: nix crassa.

4. Jan.: dies frigidissima.

22. Jan.: hat sich ain grosser stoß in der Thonau auferhept und das wasser weit in di statt gangen und biß an di weinberg, auch das Tunckau (Donaugau) ausgangen, di traidt verderpt mit anderem großen mercklichem schaden an mulen und heusern.

1507, 2. Jan.: mirabilis et nix crassa.

3. Jan.: ad noctem resolutio magna.

29. Juni: ventus vehementissimus per totam Germaniam.

1511 , hoc anno fuit mirabilis et illa incepit circa festivitatem S. Steffani et diebus sequentibus sind 33 schne auf ainander gefallen et duraverunt per longum intervallum und sind drei stoß in der thunau auf ainander kommen, die holtzen prucke weg gefurt und sonst großen schaden gethan; es sindt auch sonst übergroße und ungewondliche wasser gewest biß lang nach pfingsten von ostern an.

17. Juni: hoc die fuit miranda et timenda tempestes de grandine et pluvia ad noctem circa horam septimam incepit, grande admodum ovorum gallinarum. Et circa monasterium S. Georgii in Prifening maximum fuit damnum commissum in eodem, roß, kue, sau mit stal und roß und paren durch das closter außgefurt, di clostermauer zerrissen, 66 schuh weg und mer dann zu schreiben ist.

Item his diebus quatuor pueri in civitate Ratisponensis periculum subierunt, ains ist gestolen und verloren, ains über ain fenster ab gefallen, ains mit ain pferdt in der Thunau ertruncken und zu Stauff gefunden.

1512, 5. Juni: pluviosa tempestas.

11. Juni: malus die.

1513, 1. Jan.: pluviosissimus annus ad multos dies.

12. Jan.: hat es umb Regen geplitzt, getondert, groß windt und hagel und regen.

16. Jan.: nix crassissima. 
21. April: his duabus diebus sind di wein und di plue umb Regenspurg vom reif all erfroren.

23. April: dies frigidissima.

28. April: nix crassa apud Inglstat.

17. Aug.: quidam Wolfgangus Burgauer moritur suis exigenus est in rota. Item ad noctem mirabilis tempestas pluvia et grandine, edituus de Henckhausen et quidam alius civis ex Kalmintz in pluvialibus aquis interierunt infra Karret et Leppersdorf (nördlich von Regensburg), et plebanus de Kalmintz vix evasit. Item die weingarten ausgefletzt.

1514, 11. Jan.: nota hunc diem, quia fuit frigidissima et etate mea nulla frigidior.

19. März: dominica, videlicet Oculi, circa horam quintam ad noctem mirabilis tempestas de grandine et pluvia et fortissimis tonitruis in civitate Ratispona admodum maximae eclipsis fuitque aura prope viridi coloris.

1514, 23. Dez.: hoc anno nix primum cecidit die sabati 23 . decembris, sed statim cum magna resolutione recessit.

1516, Anno illo mirabilis tempestas in principio hiemis usque ad festum Epiphanie cum pluvia sola et circa festum S. Ursulae, nix interca tamen nulla fuit visa, sed hominibus tussiones vel tussis miranda.

1517, 27. April: illo die hat es erst gäntzlich dem wein abkert und ist alles verdorben, auch das plue alles obs, gott behuet das getraidt vor schädlich reiff.

1518, 17. Febr.: decima septima huius mensis cum sequentibus tribus diebus fuerunt frigora horrenda et admiratione digna.

24. Juni: mirabilis tempestas in principio dici de tonitruis, fulminibus et pluvia.

1519, 1. Dez.: ventus validissimus ad noctem.

1520, 1. April: die prima huius mensis, quae fuit palmtag, sindt mein schwelbel (Schwalben) kumen.

Juli: hoc anno tempore messis fuerunt tempestas de grandine horribilis in multis locis, das traid erschlagen in Bavaria.

27. Sept.: Seyberstorffer ad S. Paulum obiit mortem.

Isti duo menses fuerunt omnino pluviales et per totum maximae inundationes deinde suborta usque ad finem huius Septembris.

28. Nov.: illo die exivi ad Ingolstat propter pestem.

1522, Jan.: dies frigidissimae hoc anno illo praesertim mense.

25. Jan.: fuit ante meridiem clara et multum frigida.

25. Mai: fui illa dominica in Podmays (Bodenmais). 0 mala tempestas de pluvia ad multos dies.

8. Juni: (Pfingsten) gantz schön und warm quatuor diebus.

25. Dez.: tempus pluviale et crevit Danubius et alias inundationes magnae.

1525 ,

1528, 30. April: ultima huius mensis ad noctem mirabilis tempestas de tonitruis fortissimis et pluvia.

15. Nov.: extensissima frigiditas ad certos dies post nives maximas.

1529, 25. Juli: quo fuit festum S. Jacobi, fuerunt miserabilis tempestas de tonitruis et pluvia et fulgure, nam toties evenerunt. 


\section{Literatur}

1. AlgöWer D., Specimen hyetometriae curiosae oder Abmessung der jährlichen Regen- und Schneewässer von 1715-1721, Frankfurt am Main und Leipzig 1721

2. Bergsten T., Balthasar Hubmaier, Kassel 1961

3. Bosı K., Handbuch der Historischen Stätten Deutschlands, Band VII, Bayern, Stuttgart 1961

4. Fьoн H., Klima und Witterungsablauf in Zürich im 16. Jahrhundert, Vjschr. Naturforsch. Ges. Zürich 94 (1949)

5. Foerstu J.N., Kleine Kirchengeschichte der Stadt Regensburg, Regensburg 1946

6. Friedensburg W., Der Regensburger Convent von 1524, Historische Aufsätze, dem Andenken an Georg Waitz gewidmet, Hannover 1886

7. GMeiner C.T., Regensburger Chronik, Band 3 und 4, Regensburg 1831

8. GraU W., Antisemitismus im späten Mittelalter, Das Ende der Regensburger Judengemeinde 1450-1519, München/Leipzig 1934

9. GüNTHER S., Johann Werner aus Nürnberg und seine Beziehungen zur mathematischen und physikalischen Erdkunde, Studien zur Geschichte der mathematischen und physikalischen Geographie, 5. Heft, Halle 1878

10. Hellmann G., Repertorium der Deutschen Meteorologie, Leipzig 1883

11. Hellmann G., Neudrucke von Schriften und Karten über Meteorologie und Erdmagnetismus, Nr.13, Meteorologische Beobachtungen vom 14. bis 17.Jahrhundert, Berlin 1901

12. JANnER F., Geschichte der Bischöfe von Regensburg, 3 Bände, Regensburg 1883-1886

13. Kцемм F., Über die meteorologischen Beobachtungen des Priors Kilian Leib im Augustinerchorherrenstift Rebdorf in der Zeit vom 22.4.1513 bis 31.12.1531, Meteorolog. Rdsch. 20 (1967)

14. Lamprecht K., Deutsche Geschichte, Band 5. 1, 2.Auflage, Berlin 1896

15. LeIDINGER E., Johann Turmairs, genannt Aventinus sämtliche Werke, Band IV, München 1908

16. LENKE W., Klimadaten von 1621-1650 nach Beobachtungen des Landgrafen Hermann IV. von Hessen (Uranophilus Cyriandrus), Ber. Dtsch. Wetterdienst, Nr. 63 (Band 9), Offenbach am Main 1960

17. Lossnitzer H., Astrologie und Meteorologie, Z. angew. Meteorologie, Das Wetter 50 (1933)

18. Oefele A.F., Rerum Boicarum scriptores, T.1, Augsburg 1763

19. Reicke E., Aus dem Leben Johann Schöners, ersten Professors für Mathematik und Geographie in Nürnberg, Abh. Naturhistor. Ges. Nürnberg 17 (1907)

20. Reiter E., Kilian Leib, Fränkische Lebensbilder, Band II, herausgegeben von der Gesellschaft für Fränkische Geschichte, Eichstätt 1968

21. ReYher S., Observationes tricennales (1679-1709) circa frigus hyemale, Misc. Berolin. $I, 1710$

22. Schade O., Satiren und Pasquille aus der Reformationszeit, Band 3, Hannover 1858

23. SсноттеNLoHr K., Johann Schöner und seine Hausdruckerei, Zentralblatt für Bibliothekswesen 24 (1907)

24. SсноттеNLOHR K., Konrad Heinfogel, Ein Nürnberger Mathematiker aus dem Freundes- 
kreis Albrecht Dürers, Beiträge zur Geschichte der Renaissance und Reformation, Josef Schlecht dargebracht, München und Freising 1917

25. Sснотtenlohr K., Tagebuch-Aufzeichnungen des Regensburger Weihbischofs Peter Krafft, Reformationsgeschichtliche Studien und Texte, Heft 37, Münster i. W. 1920

26. Staber J., Kirchengeschichte des Bistums Regensburg, Regensburg 1966

27. WeIswedel W., Balthasar Hubmaier, der Vorkämpfer für Glaubens- und Gewissensfreiheit, Kassel 1939

28. WoLf R., Geschichte der Astronomie, München 1877, Neudruck 1965

29. Zinner E., Die Fränkische Sternkunde im 11.-16. Jahrhundert, Ber. Naturforsch. Ges. Bamberg XXVII, 1934

30. Zinner E., Leben und Wirken des Johannes Müller von Königsberg, genannt Regiomontanus, Schriften zur bayerischen Landesgeschichte 31, München 1938 